\title{
A prospective observational study of the safety and effectiveness of intramuscular psychotropic therapies in patients who are acutely agitated: Greek cohort results Vasiliki Psarra*1,2, Vangelis Drossinos ${ }^{1}$ and Massaras Dimitrios ${ }^{1}$
}

Address: ${ }^{1}$ Medical Department, Pharmaserve-Lilly, Greece and 2Boarding House "Erato"

* Corresponding author

from International Society on Brain and Behaviour: 3rd International Congress on Brain and Behaviour

Thessaloniki, Greece. 28 November - 2 December 2007

Published: 17 April 2008

Annals of General Psychiatry 2008, 7(SuppI I):SI75 doi:I0.II86/I744-859X-7-SI-SI75

This abstract is available from: http://www.annals-general-psychiatry.com/content/7/SI/SI75

(C) 2008 Psarra et al.; licensee BioMed Central Ltd.

\section{Background}

Agitation is an urgent condition involving motor hyperactivity and a common manifestation of various underlying psychopathological conditions, including schizophrenia and bipolar disorder [1]. The primary purpose of this observational study was to describe the occurrence of EPS and secondary to describe the course of agitation during a 24-hour period, in patients with schizophrenia and bipolar disorder with associated acute agitation treated with IM psychotropic therapies.

\section{Materials and methods}

In this multicenter, prospective, observational study were included acutely agitated patients with schizophrenia or bipolar disorder who required treatment with at least one injection of an IM psychotropic drug. Therapies were chosen by the clinician. EPS were recorded as reported episodes of acute dystonia, akathisia and Parkinsonism. The course of agitation symptoms was studied with the use of CGI-S (Clinical Global Impression of Severity), CGI-I (Clinical Global Impression of Improvement) and PANSS-EC (Positive and Negative Syndrome ScaleExcited Component). The present analysis only included patients from the Greek cohort. Patterns of prescription are described.

\section{Results}

A total of 1945 patients were included from 12 European countries, the Greek cohort consisted of 300 patients, who completed the 24-hour period. Two post-hoc therapies cohorts were analyzed: olanzapine IM (120 patients) and other IM (180 patients). EPS were described in $11.3 \%$ of the patients. $51.7 \%$ of the patients received anticholinergic medication in the 24-hour period following IM injection $(20.8 \%$ for the olanzapine IM and $72.2 \%$ for the other IM cohort). Both cohorts showed improvement in agitation symptoms at 1 and 24 hours after baseline injection as shown in the ratings of CGI-S (mean overall CGI$S$ decrease: 0.9 and 1.6 respectively), CGI-I (mean overall CGI-I: 2.7 and 2.3 respectively) and PANSS-EC (mean overall Total PANSS-EC decrease: 5.8 and 8.8 respectively).

\section{Conclusions}

IM psychotropic therapies appeared to be well tolerated for the treatment of acute agitation in patients with schizophrenia and bipolar disorder. Moreover, patients treated with IM psychotropics (olanzapine and other IM) showed improvement in agitation measures, after 1 and 24 hours.

\section{Acknowledgements}

The Hellenic ObsIM Study Group: Bouka E, Chamogeorgakis T, Charalampous A, Chaviaras I, Fokas K, Gatsonis A, Georgoulas G, Kanistras A, Karastergiou A, Karavatos A, Katsafouros K, Katsaros A, Kontis K, Manousos E, Mavreas V, Panagiotopoulou V, Skyllakos A, Sotiriou M, Stoforos $P$, Theodoropoulou S, Tsakiris F, Tzanakaki M, Tzembelikos E

\section{References}

I. Baker RW, Kinon BJ, Maguire GA, Liu H, Hill AL: Effectiveness of rapid initial dose escalation of up to forty milligrams per day of oral olanzapine in acute agitation. J Clin Psychopharmacol 2003, 23:342-348. 